\title{
Comparison of the constraint parameters in elastic-plastic fracture mechanics
}

\author{
Yu. G. Matvienko \\ Mechanical Engineering Research Institute of the Russian Academy of Science. 4 M. Kharitonievsky Per.,101990 Moscow, \\ Russia. \\ matvienko7@yahoo.com,bttp://orcid.org/0000-0003-2367-0966
}

\begin{abstract}
To describe the effect of crack-tip constraint on the stress field for finite cracked bodies, the following most widely used constraint parameters can be employed in elastic-plastic fracture mechanics, namely, local triaxiality parameter, constraint parameter $Q$, the second fracture mechanics parameter $A$ using a three-term elastic-plastic asymptotic expansion. To establish the relationship between different constraint parameters, the crack-tip stress fields are employed. Relationship between the crack-tip constraint parameters $A, A_{2}, Q$ and $\bar{S}_{p}$ of elastic-plastic fracture mechanics is investigated.
\end{abstract}

KEYWORDs. Constraint parameters; Elastic-plastic fracture mechanics.

\section{OPEN ACCESS}

Citation: Matvienko, Yu. G., Comparison of the constraint parameters in elastic-plastic fracture mechanics, Frattura ed Integrità Strutturale, 49 (2019) 36-43.

Received: 01.03 .2019

Accepted: 23.04 .2019

Published: 01.07.2019

Copyright: (C) 2019 This is an open access article under the terms of the CC-BY 4.0, which permits unrestricted use, distribution, and reproduction in any medium, provided the original author and source are credited.

\section{INTRODUCTION}

A $\mathrm{t}$ present time, it becomes obvious that classical one-parameter fracture mechanics based on traditional parameters, such as the stress intensity factor, the J-integral, etc., is not sufficient for describing the stress field in the vicinity of the crack tip for finite cracked bodies. It is necessary to take into account the crack-tip constraint effect, i.e. to employ models and criteria of two-parameter fracture mechanics. To describe the effect of crack-tip constraint in elastic-plastic two-parameter fracture mechanics, the following possible constraint parameters should be introduced into basic equations and criteria of fracture mechanics in the case of small scale yielding and large scale yielding conditions, namely, local triaxiality parameter [1], constraint parameter $\mathcal{Q}[2,3]$, the second fracture mechanics parameter $A$ of a three-term elastic-plastic asymptotic expansion [4-6]. Some aspects of the relationship between most widely used crack-tip constraint parameters have been discussed $[7,8]$.

Solutions of the parameter $A$ for 2D test specimens are obtained by extensive finite element analysis [7]. Moreover, an engineering method of estimating the second parameter $A$ in three-term elastic-plastic asymptotic expansion is developed to cover small-scale yielding to large-scale yielding loading conditions [9]. In particular, it was shown that the parameter $A$ can be calculated through the elastic non-singular T-stress in the case of small-scale yielding. Some aspects of application of the $J-A$ approach in elastic-plastic fracture mechanics as well as solutions of the parameter $A$ for 3D test specimens are discussed in Refs. $[8,10,11]$. 
The constraint parameter $Q$ in the $J-Q$ approach proposed by O'Dowd and Shih [2, 3] has been widely used in engineering applications. This parameter can be estimated as the deviation of the crack-tip stress field from that based upon possible reference fields. Relationship between the parameter $\mathcal{Q}$ and the non-singular T-stress was also established.

To quantify in-plane and out-of-plane constraints and their interaction, a unified constraint characterization parameter $A_{p}$ was proposed [12-14]. This parameter is based on the plastic area surrounded by the equivalent plastic strain isoline in the vicinity of the crack tip. A sole linear relation between the normalized fracture toughness in terms of the J-integral and $\sqrt{ } A_{p}$ was observed.

The present paper concentrates on relationship between some crack-tip constraint parameters of elastic-plastic fracture mechanics.

\section{TWO-PARAMETER $\boldsymbol{J}$ - $\boldsymbol{A}$ CONCEPT}

\section{The J-A asymptotic elastic-plastic crack-tip stress field and fracture criterion}

T $\mathrm{n}$ the case of the elastic-plastic material deformed according to the Ramberg-Osgood power-law strain hardening curve

$$
\frac{\varepsilon}{\varepsilon_{0}}=\frac{\sigma}{\sigma_{0}}+\alpha\left(\frac{\sigma}{\sigma_{0}}\right)^{n}
$$

the stress field in the vicinity of the mode I plane strain crack is described by the three-term asymptotic expansion [5, 6] (Fig. 1)

$$
\frac{\sigma_{i j}}{\sigma_{0}}=A_{0} \rho^{s} \tilde{\sigma}_{i j}^{(0)}(\theta)-A \rho^{t} \tilde{\sigma}_{i j}^{(1)}(\theta)+\frac{A^{2}}{A_{0}} \rho^{2 t-s} \tilde{\sigma}_{i j}^{(2)}(\theta)
$$

Here, $\sigma_{0} \sigma_{0}$ is the yield stress, $\alpha$ is the hardening coefficient, $n$ is the hardening exponent $(n>1), \varepsilon_{0}=\sigma_{0} / E$ is the yield strain and $E$ is Young's modulus, $J$ is the energetic integral proposed by Cherepanov [15] and Rice [16], $A$ is the second fracture parameter, $\sigma_{i j}$ are stress components $\sigma_{r}, \sigma_{\theta}$ and $\sigma_{r \theta}$ in the polar coordinate system $r \theta$ with origin at the crack tip, $\tilde{\sigma}_{i j}^{(k)}$ are dimensionless angular stress functions obtained from the solution of asymptotic problems of order (0), (1) and (2). Angular stress functions $\tilde{\sigma}_{i j}^{(0)}$ and $\tilde{\sigma}_{i j}^{(1)}$ are scaled so as maximal equivalent Mises stress is equal to unity, i.e. $\max _{\theta} \tilde{\sigma}_{e}^{(0)}=\max _{\theta} \tilde{\sigma}_{e}^{(1)}=1$. Exponent $s$ has closed form expression $s=-1 /(n+1)$. Exponent $t$ is a numerically computed eigenvalue that depends on strain hardening exponent $n$. For materials with $n=5$ and 10 that are used in this paper values of exponent $t$ are: $t(5)=0.05456, t(10)=0.06977$. Coefficient $A_{0}$ is specified as $A_{0}=\left(\alpha \varepsilon_{0} I_{n}\right)^{s}$. Dimensionless radius $\rho$ is defined by the following formula

$$
\rho=\frac{r}{J / \sigma_{0}}
$$

Comparing Eqn. (2) and the crack-tip stress field proposed by Hutchinson [17] and Rice and Rosengren [18] ((so-called the HRR field), it is easy to see that the first term of the asymptotic expansion (2) is exactly the HRR field. It can be seen that the HRR field does not describe correctly stresses in the region $1<r \sigma_{0} / J<5$ (Fig. 1) that is significant for fracture process. Finite element solutions of elastic-plastic crack problems show that the $J$ - $A$ stress field is much closer to finite element results than the HRR field [8].

The three terms of expansion (2) are controlled by two parameters, namely, the $J$-integral and the parameter $A$. The parameter $A$ is a measure of stress field deviation from the HRR field. Assuming that cleavage fracture of a specimen and a cracked structure occurs when stress fields near the crack tip are same it is possible to formulate two-parameter $J$ - $A$ fracture criterion. This assumption also justifies the use of $A$ as a constraint parameter [8]. The two-parameter fracture 
criterion implies comparison of computed $J$-integral for a cracked structure and the experimental fracture toughness $J_{C}$ corresponding to computed value of the constraint parameter $A$
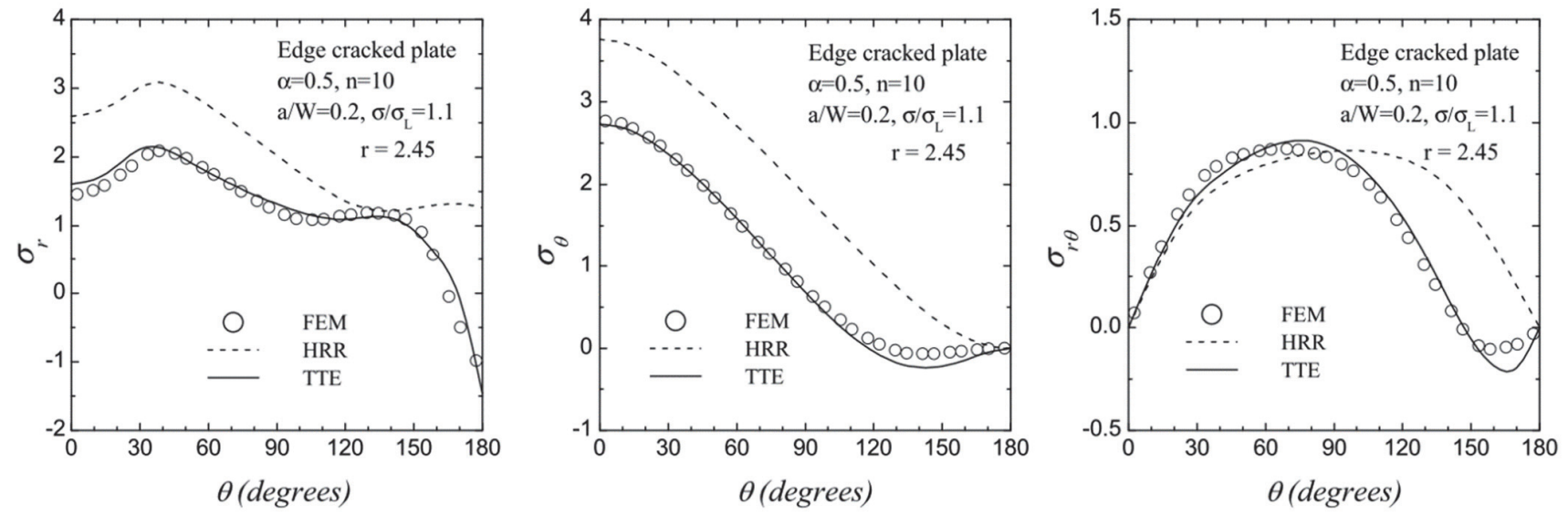

Figure 1: Comparison of the $J-A$ stress field and the HRR field for edge cracked plate.

$$
\left.J(P)\right|_{A}=J_{C}(A)
$$

Determination of the dependency $J_{C}(A)$ can be accomplished by testing cracked specimens with different constraint at fracture load. Change of the constraint conditions is achieved by varying crack length in the specimen. To avoid numerous experiments it is desirable to develop computational approaches for predicting the fracture toughness as a function of the constraint parameter $A$. Estimating change of the fracture toughness $J_{C}$ with variation of the constraint parameter $A$ can be based on an assumption that fracture under different constraint conditions corresponds to constant fracture probability $P_{f}[19]$.

\section{Numerical estimation of the J-integral and parameter $A$}

The equivalent domain integral method (EDI) $[20,21]$ is employed for computing the $J$-integral. Determination of the constraint parameter $A$ is based on stress calculation in the vicinity of the crack tip by means of the finite element method. If stresses are known at points $\left(\rho_{i}, \theta_{i}\right)$ then the value of the parameter $A$ at $i$ th point is found from the following quadratic equation [6]

$$
\begin{aligned}
& a_{i} A^{2}+b_{i} A+c_{i}=0 \\
& a_{i}=\frac{\rho_{i}^{2 t-s}}{A_{0}} \tilde{\sigma}^{(2)}\left(\theta_{i}\right), b_{i}=-\rho_{i}^{t} \tilde{\sigma}^{(1)}\left(\theta_{i}\right), c_{i}=A_{0} \rho_{i}^{s} \tilde{\sigma}^{(0)}\left(\theta_{i}\right)-\frac{\sigma^{F E M}\left(\rho_{i}, \theta_{i}\right)}{\sigma_{0}}
\end{aligned}
$$

where $\sigma^{F E M}$ is stress calculated by the finite element method. Solution of Eqn. (5) produces different $A$ values at different points due to deviation of actual stress field from the three term asymptotic expansion.

Better estimate of $A$ for the set of points is obtained by minimizing sum of squares of deviations of the $J$ - $A$ asymptotic field from the finite element results. Application of the least squares method leads to a cubic equation for the parameter $A$ [6]

$$
\begin{aligned}
& d_{3} A^{3}+d_{2} A^{2}+d_{1} A+d_{0}=0 \\
& d_{3}=2 \sum a_{i}{ }^{2}, d_{2}=3 \sum a_{i} b_{i}, \quad d_{1}=\sum\left(2 a_{i} c_{i}+b_{i}{ }^{2}\right), d_{0}=\sum b_{i} c_{i}
\end{aligned}
$$

Usually values of the stress $\sigma_{\theta}$ at finite element integration points inside region $1<\rho<4,0<\theta<45^{\circ}$ are used for estimation of the parameter $A$. 


\section{RELATIONSHIP BETWEEN THE PARAMETER $\boldsymbol{A}$ AND OTHER ELASTIC-PLASTIC CONSTRAINT PARAMETERS}

1 he most widely used constraint parameters mentioned in the introduction of this paper can be employed in engineering applications. To establish the relationship between these parameters, the crack-tip stress fields can be very useful. Relationship between the constraint parameters $A, A_{2}$ and $Q$ was discussed in Refs. [7, 8]. A constraint characterization parameter $A_{p}[12-14]$ is also analyzed in connection with the constraint parameter $A$.

\section{Relationship between the parameters $A$ and $A_{2}$}

The well-known $J-A_{2}$ approach for two-dimensional body with a mode I crack under plane strain conditions is based on the following expression of the three-term asymptotic expansion for stress in the vicinity of the crack tip in elastic-plastic material [22]

$$
\frac{\sigma_{i j}}{\sigma_{0}}=A_{1}\left[\left(\frac{r}{L}\right)^{s_{1}} \tilde{\sigma}_{i j}^{(1)}(\theta)+A_{2}\left(\frac{r}{L}\right)^{s_{2}} \tilde{\sigma}_{i j}^{(2)}(\theta)+A_{2}^{2}\left(\frac{r}{L}\right)^{s_{3}} \tilde{\sigma}_{i j}^{(3)}(\theta)\right]
$$

Here, $A_{1}=\left(\alpha \varepsilon_{0} I_{n} L \sigma_{0} / J\right)^{s_{1}}, s_{1}=-1 /(n+1)$ and $s_{3}=2 s_{2}-s_{1}$. Comparing Eq. (2) and Eq. (7), it should be noted that three corresponding dimensionless angular functions $\tilde{\sigma}_{i j}^{(k)}$ are the same in Eq. (2) and Eq. (7). Moreover, there is the connection between exponents $s_{k}$ and $t$ in Eq. (2), namely, $s_{1}=s, \mathrm{~s}_{2}=t, s_{3}=2 t-s$. Therefore, the following relationship between the constraint parameters $A$ and $A_{2}$ in the three-term asymptotic expansions of elastic-plastic crack-tip stress field can be written

$$
A_{2}=-\left(\alpha \varepsilon_{0} I_{n}\right)^{-s}\left(\frac{J}{\sigma_{0} L}\right)^{s-t} A
$$

Thus, $J-A$ and $J-A_{2}$ approaches are mathematically equivalent. The difference of these approaches is in distance scaling of $r$ according to Eqs. (2) and (7). Comparison of parameters $A$ and $A_{2}$ is shown in Fig. 2. Dependencies of both parameters


does not have its small-scale yielding value. It can be seen that the parameter $A_{2}$ tends to infinite value with load decrease. It is less convenient for constraint interpretation and engineering application.

\section{Relationship between the parameters $A$ and $Q$}

The constraint parameter $Q$ in the $J-Q$ approach $[2,3]$ is defined by the deviation of the crack-tip stress field under consideration from a reference stress field. Possible reference fields can be the HRR field or field corresponding to smallscale yielding conditions

$$
Q=\frac{\sigma_{i j}}{\sigma_{0}}-\frac{\left(\sigma_{i j}\right)_{S S Y}}{\sigma_{0}} \text { or } Q=\frac{\sigma_{i j}}{\sigma_{0}}-\frac{\left(\sigma_{i j}\right)_{H R R}}{\sigma_{0}}
$$

The following relationship between the parameters $A$ and $Q$ can be obtained from Eq. (2) and Eq. (9)

$$
Q=-A \rho^{t} \tilde{\sigma}_{\theta}^{(1)}(\theta)+\frac{A^{2}}{A_{0}} \rho^{2 t-s} \tilde{\sigma}_{\theta}^{2}(\theta) \text { at } \theta=0, \rho=2
$$

Fig. 3 presents dependencies of $A$ and $Q$ as functions of the applied load for cracks of different depth in 3 PB specimen. Constraint parameters $A$ and $Q$ demonstrate similar behavior and can be used in engineering applications. But, the $Q$ parameter can be considered as a qualitative measure of crack-tip constraint, because small errors in the crack-tip stress 
obtained by finite element method lead to the significant error in the value of $\mathscr{Q}$ [23]. The $J$ and $A$ parameters allow describing the elastic-plastic stress field near the crack tip with sufficient accuracy.
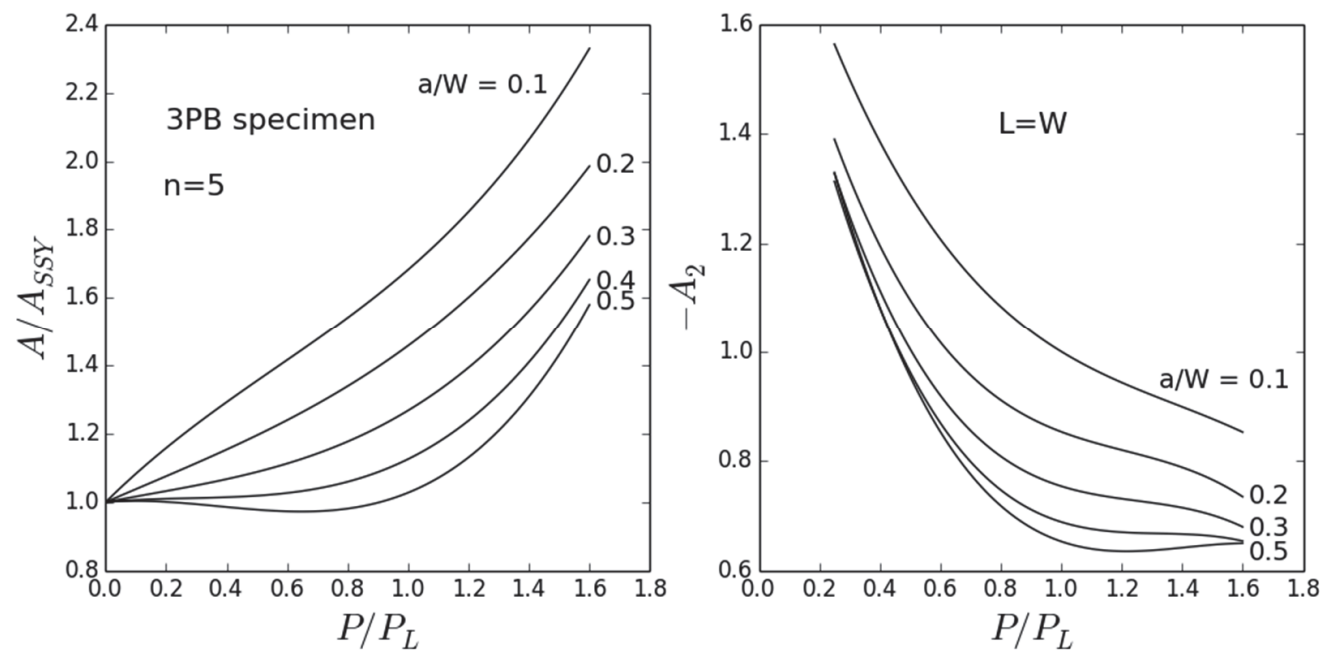

Figure 2: Comparison of the parameters $A$ and $A_{2}$ as functions of applied load $P$.
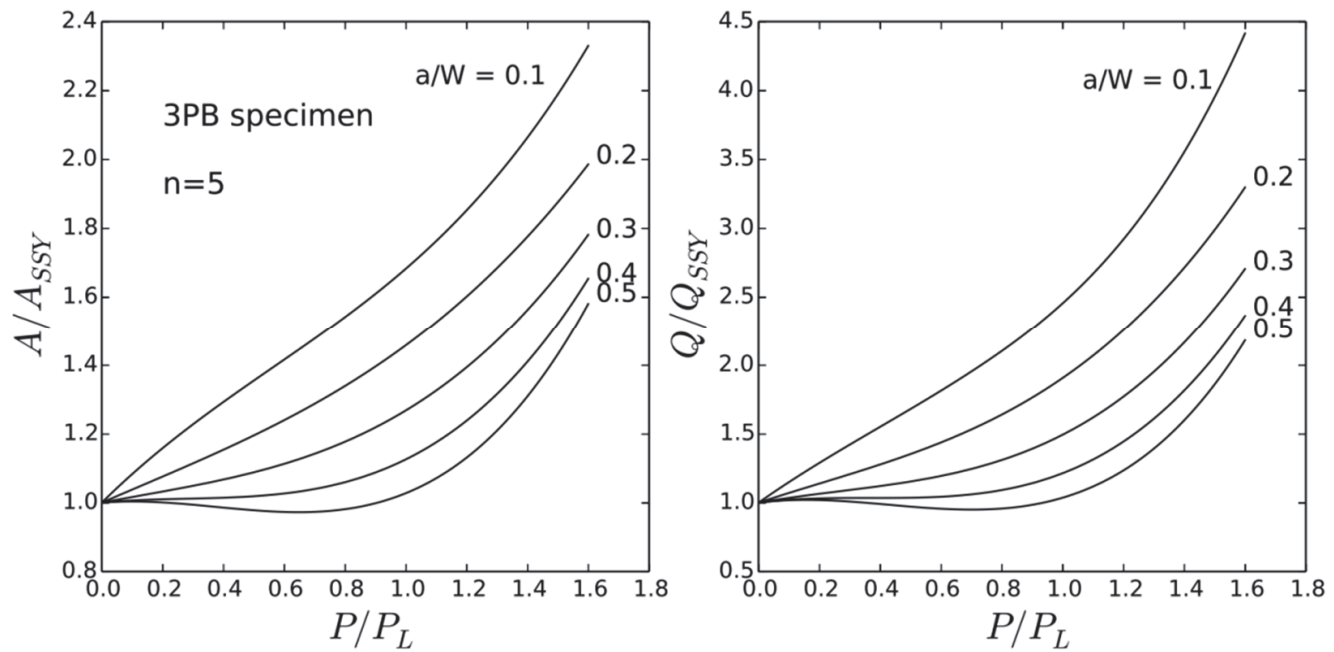

Figure 3: Comparison of the parameters $A$ and $Q$ as functions of applied load $P$.

Relationship between the parameters $A$ and $S_{p}=\sqrt{A_{p}}$

A parameter $A_{p}=A_{P E E Q} / A_{r f}$ is introduced into consideration as the unified constraint parameter to characterize inplane and out-of-plane crack-tip constraint [12-14]. The parameter $A_{P E E Q}$ is the area surrounded by the equivalent plastic strain contour with specified value of strain $\varepsilon_{p}$, the parameter $A_{r f}$ is $A_{P E E Q}$ with same strain $\varepsilon_{p}$ measured in a standard specimen test. Finite element method based on Gurson-Tvergaard-Needleman damage model was used to calculate the equivalent plastic strain $\varepsilon_{p}$ distribution ahead of the crack tip for specimens with different in-plane and out-of-plane constraints. The following plastic strains $\varepsilon_{p}=0.1,0.2$ and 0.3 were employed for determination of plastic contours. It was found that $\varepsilon_{p}=0.2$ and 0.3 provides approximately linear dependencies $J_{C} / J_{r f} \operatorname{versus} \sqrt{A_{p}}$ [13]. This is not a surprising result since the area inside specified strain $\varepsilon_{p}$ is proportional to $J^{2}$. So, the $J$-integral is compared to the quantity that is 
proportional to the $J$-integral. In our opinion the parameter $S_{p}=\sqrt{A_{p}}$ is not suitable for using as a constraint parameter because plastic zone areas and J values are proportional. To avoid direct dependence of these parameters on each other, it is proposed to replace coordinate $r$ (as well as $x$ and $y$ ) with dimensionless coordinate $\rho=r /\left(J / \sigma_{0}\right)$ [8]. In this case, the area inside equivalent plastic strain becomes dimensionless

$$
\bar{A}_{\text {PEEQ }}=A_{\text {PEEQ }} /\left(J / \sigma_{0}\right)^{2}
$$

Therefore, a normalized constraint parameter $\sqrt{\bar{A}_{p}}$ can be introduced into consideration by means of the following expression

$$
\bar{S}_{p}=\sqrt{\bar{A}_{p}}, \text { where } \bar{A}_{p}=\bar{A}_{P E E Q} / \bar{A}_{r e f}
$$

The parameter $\sqrt{\bar{A}_{p}}$ is denoted as $\bar{S}_{p}$. The modified constraint parameter $\bar{S}_{p}$ should be less dependent on the J-integral. In this case, the separation of the constraint parameter and the J-integral improves the quality of a fracture criterion that is based on these parameters.

Behavior of the parameter $\bar{S}_{p}$ as a function of $A / A_{S S Y}$ is investigated using the $J$ - $A$ elastic-plastic asymptotic field. The area surrounded by the equivalent plastic strain contour with specified value of strain $\varepsilon_{p}$ is estimated with the following algorithm [8]. Stress $\sigma_{p}$ corresponding to specified plastic strain $\varepsilon_{p}$ can be represented as

$$
\frac{\sigma_{p}}{\sigma_{0}}=\left(\alpha \frac{\varepsilon_{p}}{\varepsilon_{0}}\right)^{1 / n}=\left(\alpha \frac{E \varepsilon_{p}}{\sigma_{0}}\right)^{1 / n}
$$

The rectangular area in the vicinity of the crack tip is divided into square cells. Equivalent stress is computed at each cell by means of the $J-A$ elastic-plastic asymptotic field. If this stress is larger than $\sigma_{p}$, the cell area is added to $A_{P E E Q}$. Computation of the plastic area is performed in coordinates $x \sigma_{0} / J$ and $y \sigma_{0} / J$, so the resulting area is $\bar{A}_{P E E Q}$.

Fig. 4 summarizes the computed results for plastic zones inside plastic strain contours corresponding to $\varepsilon_{p}=0.1,0.2$ and 0.3 and relationships between parameters $\bar{S}_{p}$ and $A$ for a material with $\alpha=1$ and $n=5$. Smallest plastic zone corresponds to small scale yielding value of the constraint parameter $A\left(A_{S S Y}(n=5)=0.3803\right)$. Intermediate plastic zone is computed for the value of $\left(A_{S S Y}+A_{\max }\right) / 2$. Largest plastic zone is related to $A_{\max }$ (shown in Fig. 4$)$. It can be seen that there is a unique relationship between the constraint parameters $\bar{S}_{p}$ and $A$ in a wide range of specified values of strain $\varepsilon_{p}$.

\section{CONCLUSIONS}

$\mathrm{R}$ elationship between the crack-tip constraint parameters $A, A_{2}, Q$ and $\bar{S}_{p}$ of elastic-plastic fracture mechanics is based on analysis of the stress fields in the vicinity of the crack tip. The following conclusions can be drawn.

It was demonstrated that $J-A$ and $J-A_{2}$ approaches are mathematically equivalent. The difference of these approaches is in distance scaling $r$. But, in contrast to the parameter $A$, the parameter $A_{2}$ does not have its value under small scale yielding conditions. Constraint parameters $A$ and $Q$ show similar behavior. At the same time, it should be noted that small errors in the crack-tip stress obtained by finite element method lead to the significant error in the value of $Q$. The constraint parameter $S_{p}=\sqrt{A_{p}}$ based on the area surrounded by the equivalent plastic strain $\varepsilon_{p}$ contour ahead of the crack tip is not suitable as a crack-tip constraint parameter because plastic zone areas and $\mathrm{J}$ values are proportional. To avoid direct dependence of these parameters on each other, the constraint characterization parameter $\sqrt{A_{p}}$ is modified as 
follows $\sqrt{\bar{A}_{p}}=\bar{S}_{p}$. In this case, the separation of the constraint parameter and the $J$-integral improves the quality of a fracture criterion in two-parameter elastic-plastic fracture mechanics. It was shown that there is a unique relationship between the constraint parameters $\bar{S}_{p}$ and $A$ in a wide range of specified values of strain $\varepsilon_{p}$.
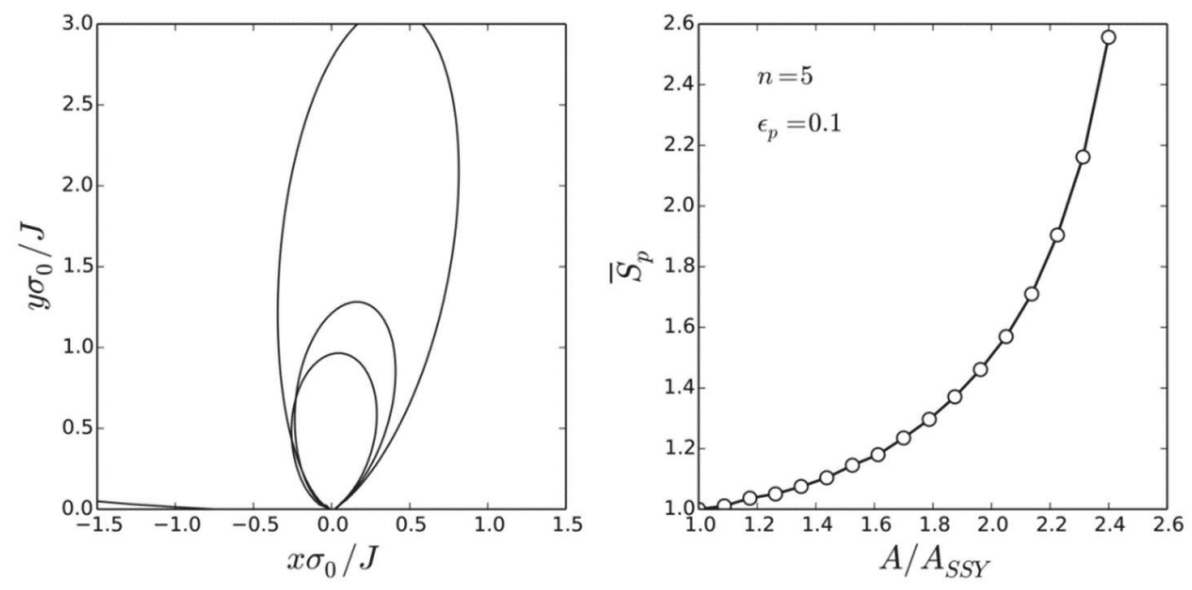

a)
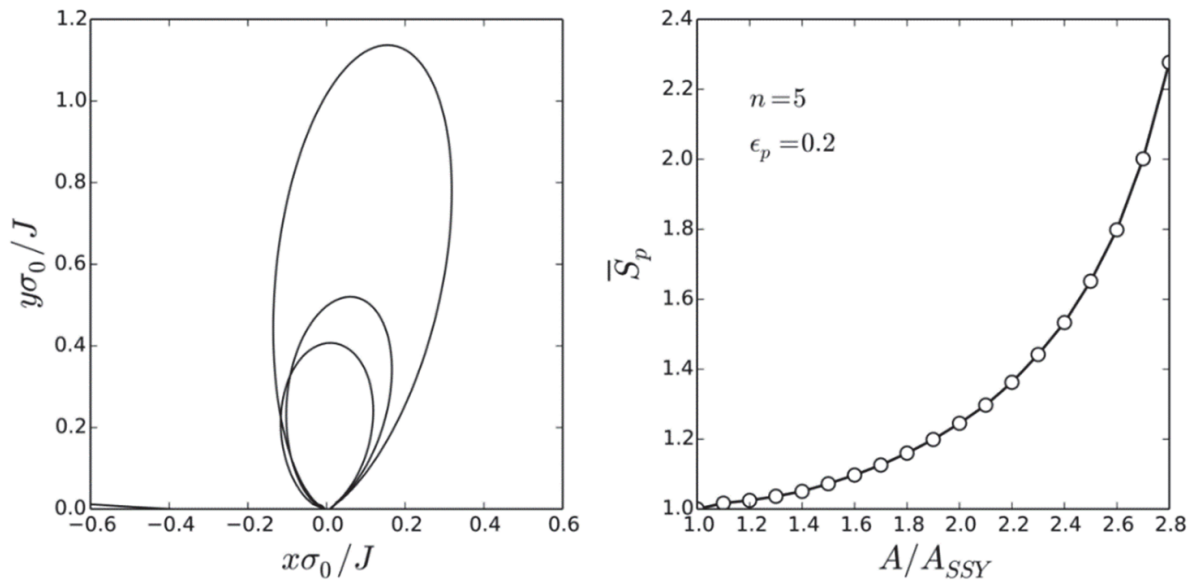

b)
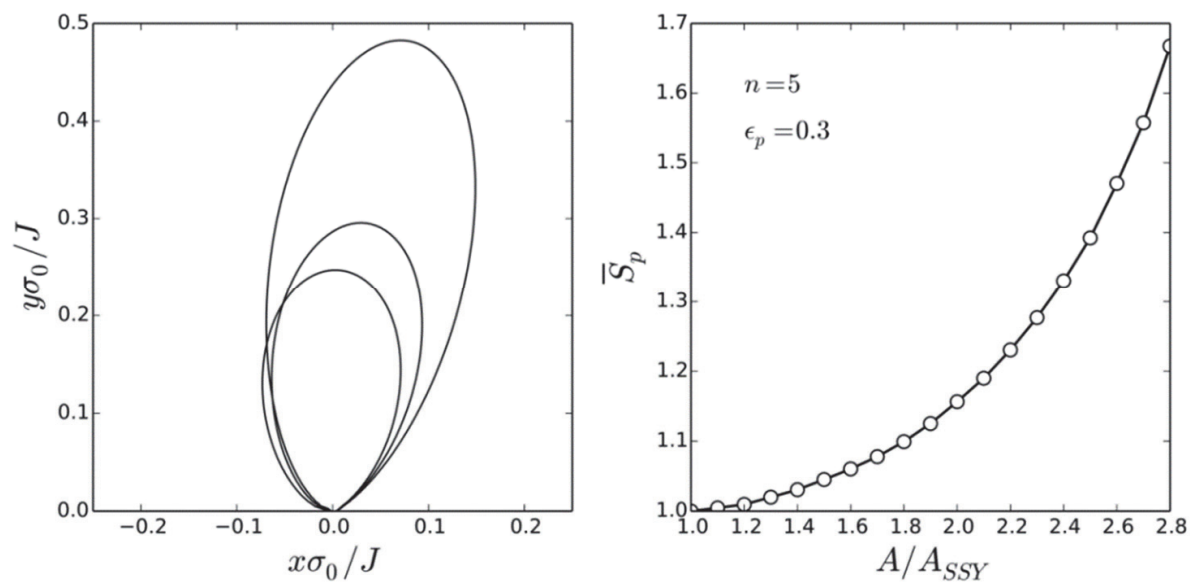

c)

Figure 4: Equivalent plastic strain isolines for $A / A_{S S Y}$ with minimal, average and maximal values (left); the constraint parameter $\bar{S}_{p}$ as a function of the parameter $A$ (right): $n=5, \varepsilon_{p}=0.1$ (a), $\varepsilon_{p}=0.2$ (b) and $\varepsilon_{p}=0.3$ (c). 


\section{ACKNOWLEDGEMENTS}

he authors acknowledge the support of the Russian Science Foundation (project N 18-19-00351).

\section{REFERENCES}

[1] Henry, B.S., Luxmoore A.R. (1997). The stress triaxiality constraint and the Q-value as ductile fracture parameter, Eng. Fract. Mechanics, 57, pp. 375-390.

[2] O’Dowd, N. P., Shih, C. F. (1991). Family of crack-tip fields characterized by a triaxiality parameter - I. Structure of fields, J. Mech. Phys. Solids, 39, pp. 989-1015.

[3] O’Dowd, N. P., Shih, C. F. (1992). Family of crack-tip fields characterized by a triaxiality parameter- II. Fracture applications, J. Mech. Phys. Solids, 40, pp. 939-963.

[4] Yang, S., Chao, Y.J., Sutton, M.A. (1993). Higher-order asymptotic fields in a power law hardening material, Eng. Fract. Mechanics, 45, pp. 1-20.

[5] Nikishkov, G.P. (1995). An algorithm and a computer program for the three-term asymptotic expansion of elasticplastic crack tip stress and displacement fields, Eng. Fract. Mechanics, 50, pp. 65-83.

[6] Nikishkov, G.P., Bruckner-Foit, A., Munz, D. (1995). Calculation of the second fracture parameter for finite cracked bodies using a three-term elastic-plastic asymptotic expansion, Eng. Fract. Mechanics, 52, pp. 685-701.

[7] Ding, Ping, Wang, Xin. (2010). Solutions of the second elastic-plastic fracture mechanics parameter in test specimens, Eng. Fract. Mechanics, 77, pp. 3462-3480.

[8] Matvienko, Yu.G., Nikishkov, G.P. (2017). Two-parameter J-A concept in connection with crack-tip constraint. Theor. Appl. Fract. Mech., 92, pp. 306-317.

[9] Ding, Ping, Wang, Xin. (2012). An estimation method for the determination of the second elastic-plastic fracture mechanics parameters, Eng. Fract. Mechanics, 79, pp. 295-311.

[10] Nikishkov, G.P., Matvienko, Yu.G. (2016). Elastic-plastic constraint parameter A for test specimens with thickness variation, Fatig. Fract. Eng. Mater. Struct., 39, pp. 939-949.

[11] Matvienko, Yu.G., Nikishkov, G.P. (2016). J-A elastic-plastic crack tip field and the two-parameter fracture criterion, Structural Integrity Procedia 2 26-33.

[12] Yang, J., Wang, G.Z., Xuan, F.Z., Tu, S.T. (2013). Unified characterisation of in-plane and out-of-plane constraint based on crack-tip equivalent plastic strain, Fatig. Fract. Eng. Mater. Struct., 36, pp. 504-514.

[13] Mu, M.Y., Wang, G.Z., Xuan, F.Z., Tu, S. T. (2015). Unified correlation of in-plane and out-of-plane constraint with cleavage fracture toughness, Theor. Appl. Fract. Mech., 80, pp. 121-132.

[14] Mu, M.Y., Wang, G.Z., Xuan, F.Z., Tu, S. T. (2017). Fracture assessment based on unified constraint parameter for pressurized pipes with circumferential surface cracks, Eng. Fract. Mechanics, 175, pp. 201-218.

[15] Cherepanov, G.P. (1967). The propagation of cracks in a continuous medium, J. Appl. Math. Mech., 31, pp. 503-512.

[16] Rice, J.R. (1968). A path independent integral and the approximate analysis of strain concentration by notches and cracks, J. Appl. Mech. ASME, 35, pp. 379-386.

[17] Hutchinson, J.W. (1968). Singular behavior at the end of a tensile crack in a hardening material, J. Mech. Phys. Solids, 16, pp. 13-31.

[18] Rice, J.R., Rosengren, G.F. (1968). Plane Strain deformation near a crack tip in a power law hardening material, J. Mech. Phys. Solids, 16, pp. 1-12.

[19] Nikishkov, G.P. (2016). Prediction of fracture toughness dependence on constraint parameter A using the weakest link model, Eng. Fract. Mech., 152, pp. 193-200.

[20] Nikishkov, G.P., Atluri, S.N. (1987). Calculation of fracture mechanics parameters for an arbitrary three-dimensional crack by the equivalent domain integral method, Int. J. Numer. Meth. Eng., 24, pp. 1801-1821.

[21] Nikishkov, G.P., Vershinin, A.V., Nikishkov, Y.G. (2016). Mesh-independent equivalent domain integral method for J-integral evaluation, Adv. Eng. Softw., 100, pp. 308-318.

[22] Chao, Y.J., Yang, S., Sutton, M.A. (1994). On the fracture of solids characterized by one or two parameters: theory and practice, J. Mech. Phys. Solids, 42, pp. 629-647.

[23] Lee, M.M.K., Boothman, D.P., Luxmoore, A.R. (1999). Effect of biaxial loading on crack driving force and constraint for shallow semi-elliptical surface flaws, Int. J. Fract., 98, pp. 37-54. 